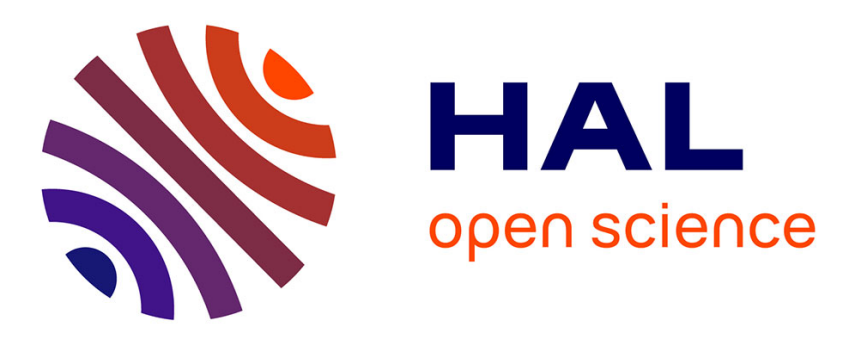

\title{
Tone Mapping Operators: Progressing Towards Semantic-awareness
}

Abhishek Goswami, Mathis Petrovich, Wolf Hauser, Frédéric Dufaux

\section{To cite this version:}

Abhishek Goswami, Mathis Petrovich, Wolf Hauser, Frédéric Dufaux. Tone Mapping Operators: Progressing Towards Semantic-awareness. IEEE International Conference on Multimedia \& Expo Workshops (ICMEW), Jul 2020, London, United Kingdom. pp.1-6, 10.1109/ICMEW46912.2020.9106057 .

hal-02543939

\section{HAL Id: hal-02543939 \\ https://hal.science/hal-02543939}

Submitted on 29 Apr 2020

HAL is a multi-disciplinary open access archive for the deposit and dissemination of scientific research documents, whether they are published or not. The documents may come from teaching and research institutions in France or abroad, or from public or private research centers.
L'archive ouverte pluridisciplinaire HAL, est destinée au dépôt et à la diffusion de documents scientifiques de niveau recherche, publiés ou non, émanant des établissements d'enseignement et de recherche français ou étrangers, des laboratoires publics ou privés. 


\title{
TONE MAPPING OPERATORS: PROGRESSING TOWARDS SEMANTIC-AWARENESS
}

\author{
Abhishek Goswami ${ }^{\star \dagger} \quad$ Mathis Petrovich WolfHauser $^{\star} \quad$ Frederic Dufaux $^{\dagger}$ \\ * DxO Labs \\ † Université Paris-Saclay, CNRS, CentraleSupélec, Laboratoire des signaux et systèmes
}

\begin{abstract}
A Tone Mapping Operator (TMO) aims at reproducing the visual perception of a scene with a high dynamic range (HDR) on low dynamic range (LDR) media. TMOs have primarily aimed to preserve global perception by employing a model of human visual system (HVS), analysing perceptual attributes of each pixel and adjusting exposure at the pixel level. Preserving semantic perception, also an essential step for HDR rendering, has never been in explicit focus. We argue that explicitly introducing semantic information to create a 'content and semantic'-aware TMO has the potential to further improve existing approaches. In this paper, we therefore propose a new local tone mapping approach by introducing semantic information using off-the-shelf semantic segmentation tools into a novel tone mapping pipeline. More specifically, we adjust pixel values to a semantic specific target to reproduce the real-world semantic perception.
\end{abstract}

Index Terms - Tone mapping, High Dynamic Range Imaging, Semantic aware exposure adjustment

\section{INTRODUCTION}

The luminance ratio between the brightest and the darkest point of a scene is called the dynamic range. In real world, this ratio is much higher than the dynamic range of most display devices. To render a High Dynamic Range (HDR) image on a Low Dynamic Range display or a photo print, a Tone Mapping Operator (TMO) has to be applied which maps the tonal values (pixel values) while compressing the dynamic range with the aim of preserving perceptual cues of the scene. Classical TMOs [1, 2, 3] utilize models based on pixel values only or incorporate features of Human Visual System (HVS) and tone map based on the perceptual attributes of each pixel.

The HVS can adapt to scenes of different dynamic ranges and still perceive semantic information. Therefore, preserving semantic perception is essential and it seems intuitive that a TMO should be aware of the semantic content of the scene. This problem has not been addressed in previous TMOs. Previous approaches have tried to preserve lightness perception,

This work has received funding from the European Union's Horizon 2020 research and innovation programme under the Marie Sklodowska-Curie Grant Agreement No. 765911 (RealVision) but have never used any information explicitly to emulate $S e$ mantic Awareness as a guide towards tonal adjustment.

In this work, we propose a new tone mapping pipeline around a probabilistic semantic framework, which guides the TMO with target lightness values for pixel specific exposure adjustment. More specifically, the proposed pipeline includes the following steps. We first decompose an image into regions (frameworks of reference) based on semantic similarity instead of only luminance distribution, using off-the-shelf semantic classifiers. We then apply tools such as matting to create soft segments (mattes) from the output of the semantic classifier. Finally, we propose to learn semantic specific lightness values for target tone modification and use them to compute semantic specific gains for each region. The two contributions of the work lie in the new semantic TMO pipeline and the methodology to learn semantic specific target lightness.

\section{RELATED WORK}

Over the past decades, TMOs have been widely studied. Functionally, TMOs are of two types: global and local. Global TMOs apply the same luminance compensation throughout the image, whereas local TMOs take into account the spatial neighborhood of each pixel. We follow a recent comparative subjective study of several classical TMOs provided by Cerda-Company et al. [4] to understand the performance of different TMOs. Kim et al. [2] (rated highly over subjective experiments [4]) propose a global TMO based on the luminance adaptation of human visual cortex. They suggest that human visual sensitivity is adapted to the average log luminance of the scene and that it follows a Gaussian distribution.

As a local approach, Krawczyk et al. [1] propose a TMO based on a probabilistic model of lightness perception (rated highly over subjective experiments [4]). They decompose an HDR image into areas of consistent luminance (lightness framework) and map each framework by adjusting the perceived 'white' point based on Gilchrist's anchoring rule [5]. They follow Durand et. al's [3] approach of smoothing the base layer and penalise local variations in the probabilistic framework to preserve local contrast. The pixel precise framework allocation based on luminance helps overcome distortions like halos. 

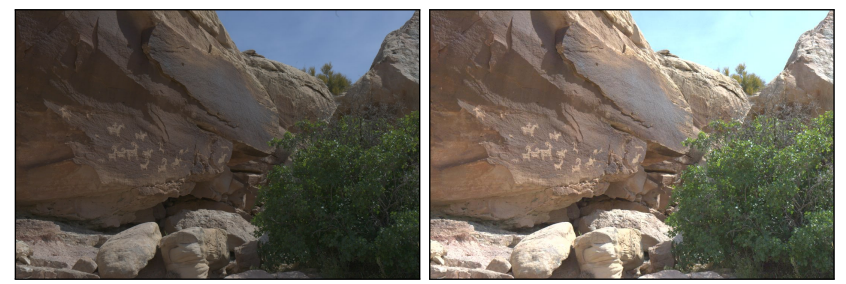

Fig. 1. Gamma-corrected HDR content Petroglyphs (left) [6] and the tone mapped image using KrawczykTMO [7] (right).

However, the idea that the HVS breaks a scene down on the basis of consistent luminance has shortcomings. We postulate that consistent luminance should not necessarily mean allocation to same framework, especially if the luminance is encountered in a different semantic context.

Fig. 1 presents the Petroglyphs image from the Fairchild HDR dataset [6]. In this image, the bright rocks on the bottom left and the sky on the top right have the same luminance. After tone mapping using Banterle's [7] implementation of KrawczykTMO [1], the rocks are no longer underexposed, but the sky seems to be overexposed. As parts of the rocks and the sky fall in the same lightness framework, both semantics get treated uniformly. We suppose that the HVS also breaks a scene down based on the semantic consistency rather than only consistent luminance. Hence we propose to create semantic frameworks instead and adjust them towards a target.

Krawczyk et. al [1] sets the white point as a local anchor inside each framework and adjusts exposure based on this anchor. Renowned photographer Ansel Adams claimed that the global average perceived luminance of mid-grey for photography is $18 \%$ of visible light [8], which has been the anchor for many TMOs. Our hypothesis is that every semantic content has a target lightness (perceived luminance) which should be reproduced by the TMO. More specifically, HDR images need to be tone mapped towards target lightnesses which are a function of the semantic label of a region in the scene and each region should be adjusted accordingly.

Recent data-driven approaches use neural networks for HDR tone mapping. Unlike classical methods, learning based TMOs do not follow an explicit model based on pixel values or human vision. They tend to learn the tone curve from a high dimensional feature representation based on training images. Rana et al. [9] follow such a data-driven approach and propose a deep learning based parameter-free TMO. Such approaches might implicitly incorporate semantic attributes but do not explicitly use semantic information.

In this paper, our objective is to explicitly define a semantic-specific target based on the content observed in the image and its luminance perception in the real world. We then guide the exposure adjustment towards that target lightness for each semantic framework. To create semantic frameworks, we use available off-the-shelf semantic segmentation methods. Since 2012, the rise of neural networks and deep learning has given us multiple models and various annotated datasets to learn and predict pixel wise semantic labels $[10,11,12,13]$. Neural networks used for semantic segmentation include PSPNet [14], DeepLab [15, 16] and FastFCN [17]. In this work, we use these available tools as a black box to learn semantic-specific target lightness values and to segment images into semantic frameworks. Our contribution is how we use such semantic frameworks to guide the exposure adjustment, thus making the TMO semantic-aware.

\section{PROPOSED TONE MAPPING SYSTEM}

We propose a novel tone mapping system for decomposing an HDR scene into frameworks of reference and then adjusting the exposure of each framework based on its content. Fig. 2 presents an overview of our proposal. Each module is discussed in more details hereafter.

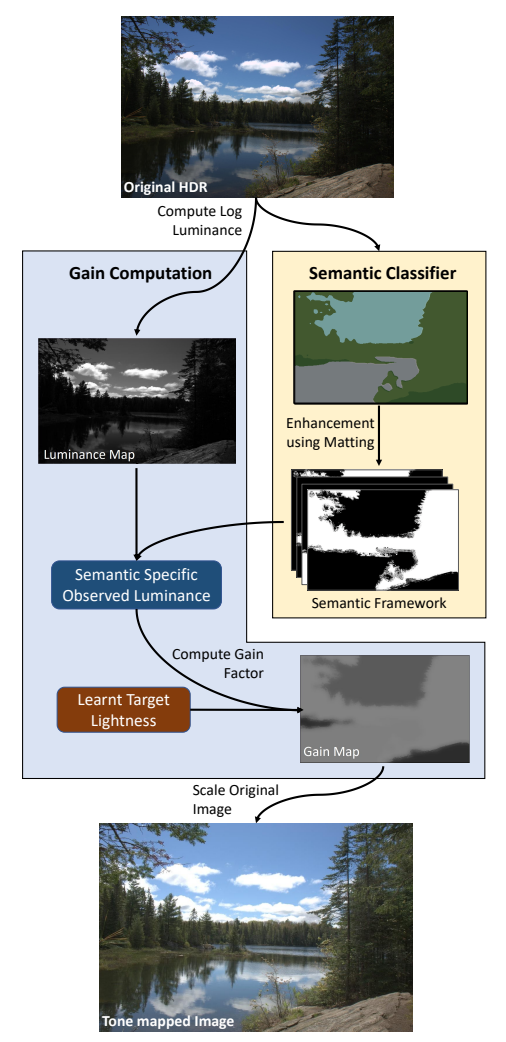

Fig. 2. Proposed tone mapping pipeline.

\subsection{Semantic classifier}

\subsubsection{Semantic Segmentation}

The first step is to create our semantic frameworks. We start with the original HDR image, resized by a factor of 4 on each dimension to reduce memory requirements and computational complexity. We classify the image into semantic labels, 

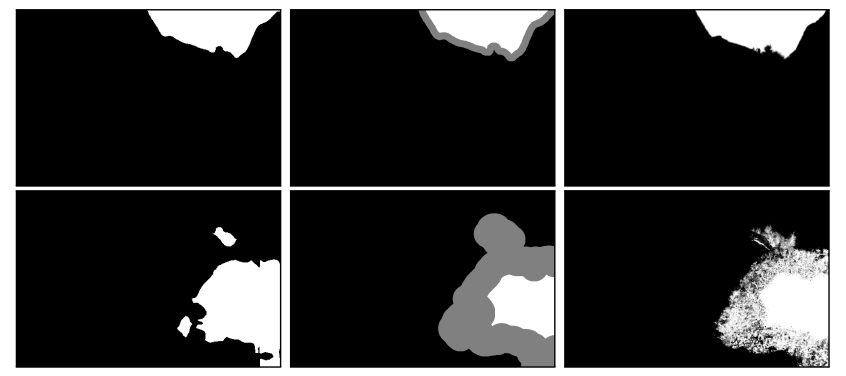

Fig. 3. Trimaps (middle) and mattes (right) for sky (top) and vegetation (bottom) for the image Petroglyphs (Fig.1).

using available deep learning tools. We experimented with existing models such as PSPNet, Deeplabv3, Deeplabv3+ and FastFCN trained over annotated datasets (Pascal VOC, Cityscapes and ADE20K). We chose FastFCN architecture pretrained over ADE20K dataset due to the relatively better inference and wider range of labels (150 in total) [17].

Our hypothesis is that, the HVS analyses a scene by breaking it down into regions of semantic similarity and that the perceptual cues from these regions are treated similarly. It should be noted that the semantic distance between the 150 labels in ADE20K is not equal. Dogs and cats, for example, are different semantic labels, but when adjusting exposure, the HVS would probably consider them semantically similar.

Compiling an exhaustive set of semantically similar classes is a non-trivial problem and needs further research beyond the scope of this paper. Based on the contents of the images in our dataset (discussed in Sec. 3.2.1) we have heuristically defined 9 semantically similar classes, which require unique lightness adjustment. The 9 semantic classes are: sky, mountain, vegetation, water, human subject, still-life subject, city, indoor, others. Consequently, we grouped the 150 labels from ADE20K into these 9 predefined classes. For e.g. labels such as sea, river, lake etc. were mapped to the class water. The labels obtained for our image from FastFCN are merged according to our predefined classes to compute one binary map for each semantic class observed. The next step is to enhance the pixel precision of these binary maps.

\subsubsection{Proposed enhancement using matting}

Obtaining a pixel precise semantic map is a non-trivial problem. FastFCN results in imprecision in the high frequencies such as object boundaries. While making pixel specific adjustments, such imprecision can lead to distinctive distortions. Therefore, we require approaches such as soft segmentation to deal with imprecise semantic label allocation.

$\mathrm{Hu}$ et al. [18] proposed a two-step enhancement using the output of a semantic classifier and applying matting to it. Aksoy et al. [19] have considered the problem from a spectral segmentation viewpoint and used high and low level image features to obtain a soft segment. Learning based methods
[20] also compute soft segments but they seem to associate certain semantic classes (such as vegetation and sky) systematically with the background and hence are unable to provide generalized semantic masks suitable for our purpose.

After experimenting with various matting techniques, we selected Alpha matting [21], due to the fewer artifacts leading to better quality while blending differently exposed segments. We first generate a morphologically expanded region of uncertainty called the trimap around the boundary of the semantic segment. Instead of binary labels, Alpha matting then provides fuzzy labels between $[0,1]$ to each uncertain pixel denoting the probability of it belonging to that segment.

We notice that the map corresponding to semantic class vegetation is likely to produce segments with finer local contrast at their borders. Conversely, maps for other semantic classes such as sky have lower contrast borders. So a thicker trimap border for the former and a relatively thinner border for the latter yields better results (See Fig. 3). The Alpha matting returns one soft segmented matte for each observed class where each pixel has a fuzzy label i.e. a probability of belonging to that matte. The mattes may overlap at boundaries. Consequently, we normalize the label values at each pixel location over all mattes to get class-specific probability for that pixel. This collection of normalized mattes, called semantic framework shows, for every pixel $p_{x, y}$ in the image, its belongingness $P_{x, y, i}$ to each framework $\mathcal{F}_{i}$.

\subsection{Gain Computation}

\subsubsection{Learning target lightness}

The next objective is to use the semantic framework to adjust the observed luminance of the original image towards a target lightness. One of the main contributions of this work is a new methodology to learn semantic-specific perceived luminance. We propose to learn the target lightness (perceived luminance) for different semantic classes from a real-world dataset of well-exposed images. To this aim, we have created a dataset of 830 high resolution [4000 $\times 3000]$ LDR images from freely available sources [22].

More precisely, we compute a luminance histogram for each of our 9 classes over the entire dataset. Fig. 4 shows three such histograms, as well as the world histogram (which contains all pixels in our dataset). Ideally, semantics with different perceived luminance should have different histograms and hence should require different classification and exposure adjustment. This hypothesis holds for our 9 classes. Since the semantic segmentation is not pixel precise, we choose the median of the histogram to compute the class specific target lightness, for its robustness against outliers.

Table 1 shows the target lightness for our 9 classes. Intuitively, some semantics like sky are expected to have a brighter target, while others, a combined class for unrelated labels, has a target close to the global mid-grey of $18 \%$. The class human has a surprisingly low target, which can be explained by the 


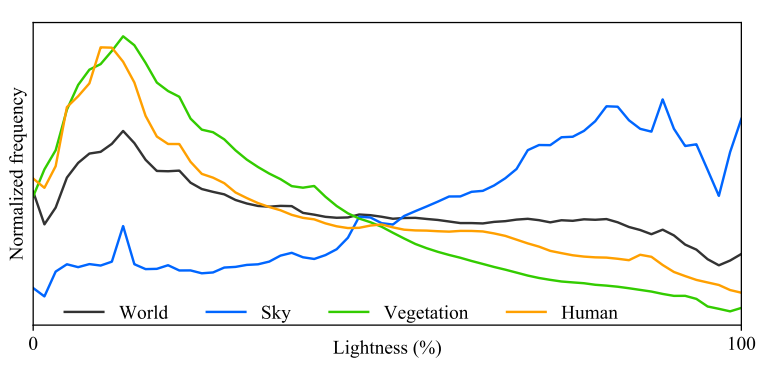

Fig. 4. Histogram of luminance values observed in our dataset, for different semantic classes. Sky and vegetation are significantly different from the world average.

fact that the semantic classifier does not differentiate between skin and non-skin part of humans.

\subsubsection{Computing gain factors}

Next, we compute the luminance map, $L_{x, y}$ for our test image using weighted average of the linear $R G B$ values. Using the semantic framework and a probability threshold, we get a luminance distribution for each matte framework. We consider the luminance values of only those pixels which have a belongingness above a threshold $T_{b}$, empirically set to 0.8 . The median of this luminance distribution gives us the observed luminance $L_{o b s(i)}$ for each matte framework. The target lightness $L_{t a r(i)}$ is learnt from our dataset, as detailed in Table 1. Using the two values, we compute a class specific gain factor, $\gamma_{i}$. Finally, we merge the gain factors weighted by the pixel belongingness in the semantic framework to compute a gain map of the same dimension as of the input image. Every in$\operatorname{dex}(x, y)$ in this gain map represents the pixel specific gain factor $\Gamma_{x, y}$ for tone mapping.

$$
\begin{aligned}
\gamma_{i} & =\frac{L_{\operatorname{tar}(i)}}{L_{o b s(i)}} \\
\Gamma_{x, y} & =\sum_{\mathcal{F}_{i}} \gamma_{i} \cdot P_{x, y, i}
\end{aligned}
$$

In order to preserve local contrast and details at transition boundaries, we use spatial information of the pixel neighborhood while computing the gain factors. More precisely, we apply a bilateral filter [23] to the mattes while creating the semantic framework to penalise for local variations. Finally, the gain map is used to scale the original image pixel by pixel to obtain our final tone mapped image.

\section{RESULTS}

In this section, we analyse the performance of our proposed tone mapping algorithm. Fig. 5 presents three HDR images from the Fairchild dataset and their respective tone mapped LDR images using KrawczykTMO [1], KimKautzTMO [2], and our SemanticTMO. We aim to analyze the results based

\begin{tabular}{|c|c|c|}
\hline & \multicolumn{2}{|c|}{ Target Lightness } \\
Label & sRGB (\%) & Linear (\%) \\
\hline sky & 72 & 48.5 \\
mountain & 36 & 10.5 \\
vegetation & 27 & 5.6 \\
water & 42 & 14.8 \\
human subject & 29 & 6.5 \\
still-life subject & 32 & 8.2 \\
city & 43 & 15.6 \\
indoor & 36.5 & 10.8 \\
others & 43 & 15.6 \\
\hline
\end{tabular}

Table 1. Target lightness for different semantic classes.

on three factors: Exposure compensation, Aesthetic presentation and Distortions.

Exposure compensation: It is straightforward to notice the different gains achieved by KrawczykTMO, KimKautzTMO and SemanticTMO. KrawczykTMO and KimKautzTMO enhance shadows based on the luminance distribution only. The green bush and surrounding rocks in Petroglyphs are assigned positive gains but their relative distance in the original luminance histogram is not maintained after compression, leading to loss of relative contrast. Same goes for the shadows on the ground in Jesse's Cabin. SemanticTMO treats the shadows on the basis of luminance and the semantic map, thereby preserving relative contrast. Hence, images tone mapped with SemanticTMO are not as washed out or flat as the others.

Aesthetic representation: Aesthetic quality, though subjective, can be discussed on the basis of colour representation and preservation of photographic intent. We observe the representation of sky in all the images. SemanticTMO provides better colour and contrast representation than KimKautzTMO and KrawczykTMO. Manual photo-retouching tends to enhance primary subjects even if that requires suppressing background regions or shadows. Using SemanticTMO, regions of shadows are enhanced but moderately to preserve the global perceptual attributes of the primary subjects, such as the cabin and the hall dome. This is possible due to the inclusion of semantic information, as the TMO determines the gain as a function of the target lightness for each semantic framework.

Distortions: TMOs should compress the dynamic range without introducing distortions. KrawczykTMO and KimKautz$T M O$ rate highly in this aspect. SemanticTMO introduces some distortions in its current implementation, due to shortcomings of the FastFCN semantic classifier. Precise semantic segmentation is an ill-posed problem. Results can be inconsistent and poor when handling translucent or complex structures. Pixel-precision is not guaranteed even with matting. The Petroglyphs image is an example of pixel-precise mask without halos but the Cabin image shows limitations due to the dense distribution of vegetation in sky region.

In order to supplement the above observations, we also score the tone mapped images using two recent Image quality 

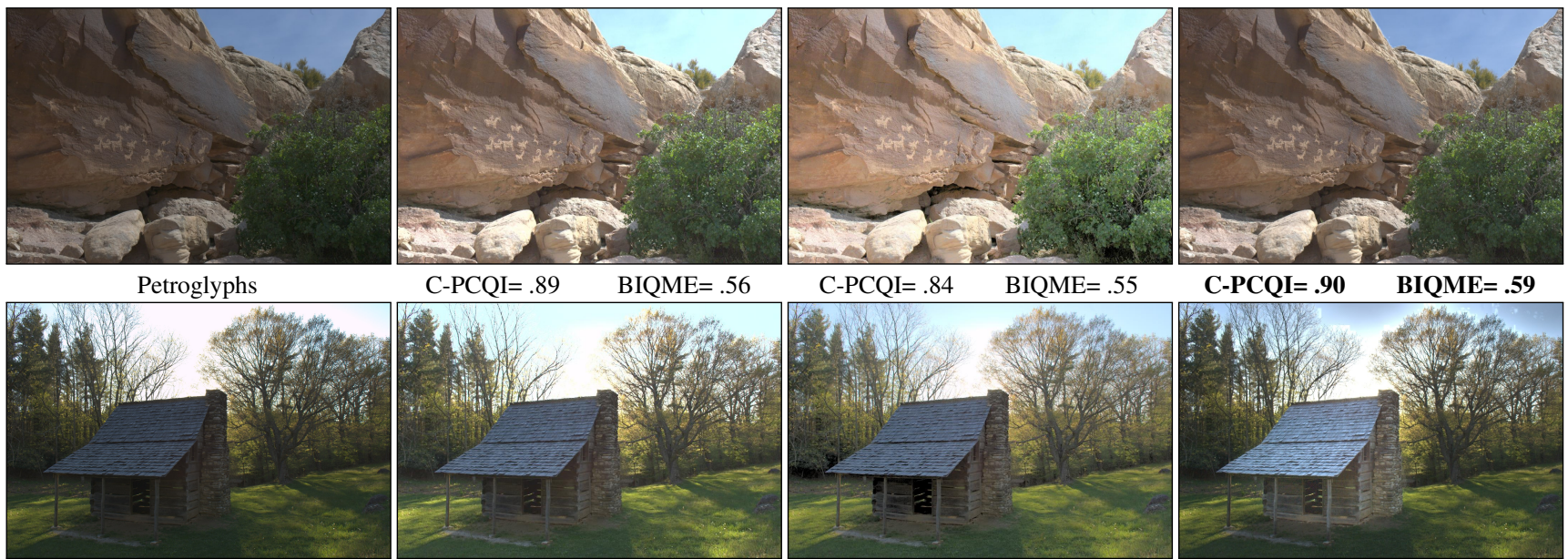

$\mathrm{C}-\mathrm{PCQI}=89$

$\mathrm{C}-\mathrm{PCQI}=.84$

$\mathrm{BIQME}=.55$
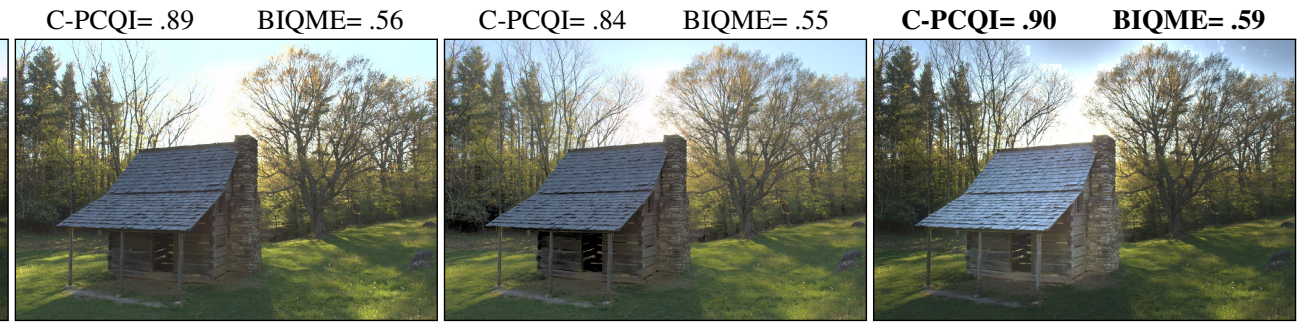

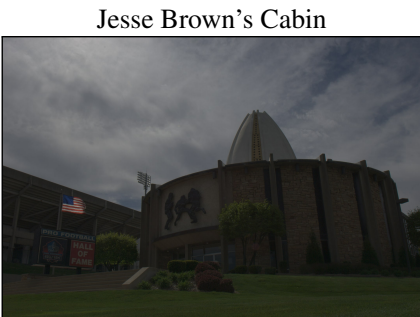

Hall of Fame $\mathrm{C}-\mathrm{PCQI}=.79 \quad \mathrm{BIQME}=.58$ $\mathrm{C}-\mathrm{PCQI}=.78 \quad \mathrm{BIQME}=.58$

$\mathrm{C}-\mathrm{PCQI}=.83 \quad \mathrm{BIQME}=.63$

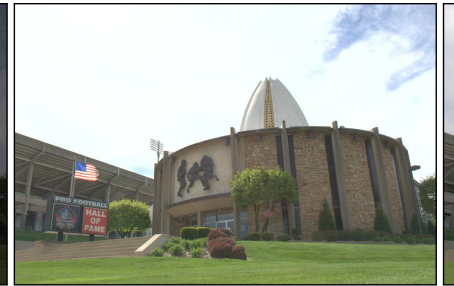

$\mathrm{C}-\mathrm{PCQI}=.68$

$\mathrm{BIQME}=.45$

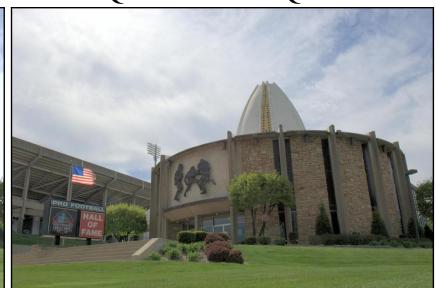

$\mathrm{C}-\mathrm{PCQI}=.77 \quad \mathrm{BIQME}=.56$

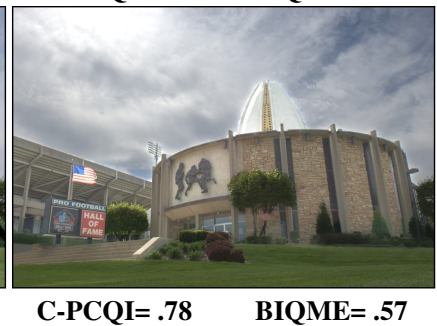

Fig. 5. Left: Gamma corrected HDR images from Fairchild HDR Dataset and respective tone mapped images using KrawczykTMO [1] \& KimKautzTMO [2] (implemented using HDR toolkit by Banterle [7]) and the proposed SemanticTMO. C-PCQI and $B I Q M E[24]$ scores have been computed for each tone mapped image and the best scores have been highlighted.

assessment (IQA) metrics. C-PCQI [24], a full reference metric, measures the quality for contrast enhanced images with importance on colourfulness. BIQME [24], a no reference metric, considers five influencing factors, contrast, sharpness, brightness, colorfulness and naturalness of images, contributing towards image quality and extracts a total of 17 features to assign a score to the tone mapped image. For both of these metrics, a higher score implies better image quality. We observe in Fig. 5, that our SemanticTMO consistently performs better than its two competitors on the test images.

\section{CONCLUSION AND FUTURE WORK}

In this paper, we discussed the limitations of existing TMOs and the benefits of explicitly including semantic information. We proposed a TMO, where we created a probabilistic semantic framework for each scene using matting and adjusted the exposure of each semantically similar region according to the semantic framework. Consequently, we created a database to learn the target lightness of different semantic classes.

The IQA scores from Fig. 5 show that our SemanticTMO performs better than KimKautzTMO and KrawczykTMO on the test images. This positively reinforces our hypothesis that different semantic classes require different target values and that introducing semantic awareness in the tone mapping process helps preserve the perception of the HDR image. Therefore, in this paper, we have shown that semantic awareness can improve upon existing TMOs.

Our work still has some limitations and can be further improved in several aspects. First, semantic segmentation is not as precise as luminance-based segmentation and can be refined further. Second, our dataset to learn target lightness is fairly small. A larger dataset can lead to a more robust scene classification to find semantically similar key classes for real world data. The median is not a statistically ideal choice to compute the target lightness and hence, the luminance distribution should rather be taken into account. Another important limitation is the non-availability of annotated training data for photographically meaningful semantic classes. The class human subject, for example, makes perfect sense for autonomous systems, but is almost meaningless for adjusting exposure. Skin and skin type would be more helpful.

This paper opens several avenues of future research. We aim to conduct subjective assessment of our results. We do not handle the scenario when a semantically uniform region receives non-homogeneous illumination. In future, we aim to create a hybrid approach based on both luminance and semantic similarity. 


\section{REFERENCES}

[1] G. Krawczyk, K. Myszkowski, and H.P. Seidel, "Lightness perception in tone reproduction for high dynamic range images," in The European Association for Computer Graphics 26th Annual Conference EUROGRAPHICS 2005. vol. 24, Blackwell.

[2] M. H. Kim and J. Kautz, "Consistent tone reproduction," in Proceedings of the Tenth IASTED International Conference on Computer Graphics and Imaging. ACTA Press Anaheim, CA, USA, 2008, pp. 152-159.

[3] F. Durand and J. Dorsey, "Fast bilateral filtering for the display of high-dynamic-range images," in ACM transactions on graphics (TOG). ACM, 2002, vol. 21, pp. 257-266.

[4] X. Cerdá-Company, C. A. Párraga, and X. Otazu, "Which tone-mapping operator is the best? A comparative study of perceptual quality," CoRR, vol. abs/1601.04450, 2016.

[5] A. Gilchrist, C.s Kossyfidis, F. Bonato, T. Agostini, J. Cataliotti, X. Li, B. Spehar, V. Annan, and E. Economou, "An anchoring theory of lightness perception," Psychological review, vol. 106, pp. 795-834, Nov. 1999.

[6] M.D. Fairchild, “The hdr photographic survey," pp. 233-238, Jan. 2007.

[7] F. Banterle, A. Artusi, K. Debattista, and A. Chalmers, Advanced High Dynamic Range Imaging (2nd Edition), AK Peters (CRC Press), Natick, MA, USA, July 2017.

[8] B. Brown, Cinematography: Theory and Practice : Imagemaking for Cinematographers, Directors \& Videographers, Focal Press, 2002.

[9] A. Rana, P. Singh, G. Valenzise, F. Dufaux, N. Komodakis, and A. Smolic, "Deep Tone Mapping Operator for High Dynamic Range Images," IEEE Transactions on Image Processing, vol. 29, no. 1, pp. 12851298, Dec. 2019.

[10] M. Everingham, S. M. Eslami, L. Gool, C. K. Williams, J. Winn, and A. Zisserman, "The pascal visual object classes challenge: A retrospective," Int. J. Comput. Vision, vol. 111, no. 1, pp. 98-136, Jan. 2015.

[11] R. Mottaghi, X. Chen, X. Liu, N. Cho, S. Lee, S. Fidler, R. Urtasun, and A. Yuille, "The role of context for object detection and semantic segmentation in the wild," in Proceedings of the 2014 IEEE Conference on Computer Vision and Pattern Recognition. pp. 891-898, IEEE Computer Society.

[12] B. Zhou, H. Zhao, X. Puig, S. Fidler, A. Barriuso, and A. Torralba, "Scene parsing through ade20k dataset," in Proceedings of the IEEE conference on computer vision and pattern recognition, 2017, pp. 633-641.

[13] M. Cordts, M. Omran, S. Ramos, T. Rehfeld, M. Enzweiler, R. Benenson, U. Franke, S. Roth, and B. Schiele, "The cityscapes dataset for semantic ur- ban scene understanding," in Proceedings of the IEEE conference on computer vision and pattern recognition, 2016, pp. 3213-3223.

[14] H. Zhao, J. Shi, X. Qi, X. Wang, and J. Jia, "Pyramid scene parsing network," CoRR, vol. abs/1612.01105, 2016.

[15] L. Chen, G. Papandreou, F. Schroff, and H. Adam, "Rethinking atrous convolution for semantic image segmentation," 2017.

[16] L. Chen, Y. Zhu, G. Papandreou, F. Schroff, and H. Adam, "Encoder-decoder with atrous separable convolution for semantic image segmentation," in Proceedings of the European conference on computer vision (ECCV), 2018, pp. 801-818.

[17] H. Wu, J. Zhang, K. Huang, K. Liang, and Y. Yu, "Fastfcn: Rethinking dilated convolution in the backbone for semantic segmentation," 2019.

[18] G.G Hu and J.J. Clark, "Instance segmentation based semantic matting for compositing applications," 2019.

[19] Y. Aksoy, T.H. Oh, S. Paris, M. Pollefeys, and W. Matusik, "Semantic soft segmentation," ACM Transactions on Graphics (TOG), vol. 37, no. 4, pp. 72, 2018.

[20] N. Xu, B. Price, S. Cohen, and T. Huang, "Deep image matting," in Proceedings of the IEEE Conference on Computer Vision and Pattern Recognition, 2017, pp. 2970-2979.

[21] E. S. L. Gastal and M. M. Oliveira, "Shared sampling for real-time alpha matting," Comput. Graph. Forum, vol. 29, pp. 575-584, 2010.

[22] "unsample.net powered by unsplash!," https://unsplash.com/, http://unsample.net/, Accessed: 2019-09-15.

[23] C. Tomasi and R. Manduchi, "Bilateral filtering for gray and color images.," in Iccv, 1998, vol. 98, p. 2.

[24] K. Gu, D. Tao, J.F. Qiao, and W. Lin, "Learning a noreference quality assessment model of enhanced images with big data," IEEE transactions on neural networks and learning systems, vol. 29, no. 4, pp. 1301-1313, 2017. 